\title{
Logarithmic singularity in the surface free energy near commensurate-incommensurate transitions
}

\author{
Weixiong Li and Hyunggyu Park $†$ \\ Department of Physics, Carnegie-Mellon University, Pittsburgh, PA 15213, USA
}

Received 23 July 1990

\begin{abstract}
We study finite-size-scaling behaviour of a two-dimensional random tiling model. This model exhibits commensurate-incommensurate phase transitions of the PokrovskyTalapov type where anisotropic scaling appears. A simple scaling argument near this transition suggests an integral surface critical exponent which, in turn, implies a logarithmic singularity with a universal coefficient in the surface free energy. We confirm the existence of this singularity numerically employing the transfer matrix method in the case of free boundary conditions.
\end{abstract}

Finite-size corrections to the free energy have been investigated extensively in recent years, particularly after the discovery that many two-dimensional critical systems are conformally invariant [1]. Conformal invariance relates the universality class of a system to the coefficient of the second-order correction to the free energy $[2,3]\left(\mathcal{O}\left(1 / N^{2}\right)\right.$ term where $N$ is system size). For a system with surfaces (non-gauge-invariant boundary conditions), there also arises the first-order correction whose coefficient represents the surface free energy. Relatively little is known about this surface term, partly because the surface free energy is not a universal quantity [4]. Surface effects are particularly important for systems undergoing continuous phase transitions as the correlation length becomes long-ranged near criticality. Some qualitative features of surface effects were explained in the context of mean field theory [5]. Surfaces may order at the same time or even before the bulk orders, and a set of surface critical exponents associated with surface singularities emerges. Such singularities are not of great concern because in the thermodynamic limit the bulk singularities will finally dominate. However, in numerical calculations on finite sizes, they make finite-size-scaling (Fss) analysis difficult. So it is important to understand the surface term as well as the universal scaling term (the second-order term).

Recently we studied Fss behaviour of a random tiling model [6]. The model consists of parallelograms and squares with chemical potential $\tilde{\mu}$ and 0 , respectively (figure
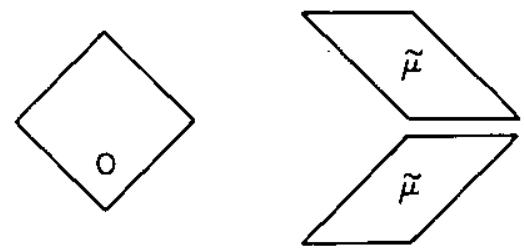

Figure 1. The tiles and their chemical potentials.

$\uparrow$ Present address: Department of Physics, Boston University, Boston, MA 02215, USA. 
1). This model forms a special case of a more general random tiling model which at proper concentrations is able to possess 8 -fold rotational symmetries, and thus may be a proper model for studying two-dimensional 8 -fold symmetric quasicrystals $[7,8]$. Using Bethe ansatz, we solved this model exactly on semi-infinite strips with periodic boundary conditions in our previous paper [6]. For $\tilde{\mu}<-\ln 2$ we have a crystal phase with purely squares, while for $\tilde{\mu}>-\ln 2$ an incommensurate phase emerges with finite domain-wall (parallelogram) densities (see figure 2 ). Domain walls run vertically on average. Near $\tilde{\mu}_{\mathrm{c}}=-\ln 2$ the domain-wall density $d$ vanishes and the heat capacity $c$ diverges with exponent $1 / 2$.

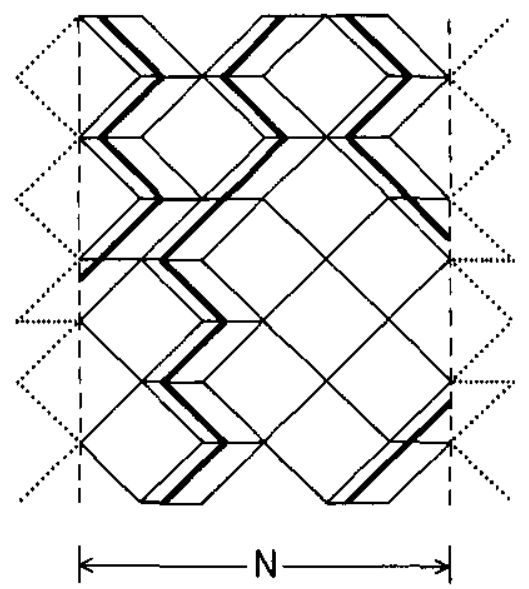

Figure 2. A typical configuration of the tiling model on a semi-infinite strip with free boundary conditions. Parts of tiles outside of the strip are cut off. Bold lines represent domain walls which are constructed by connecting centres of parallel horizontal edges. Domain walls can enter or exit at the boundary.

In this paper we concentrate on the scaling region near $\tilde{\mu}_{c}=-\ln 2$. The transition from the incommensurate (IC) to the commensurate (c) phase (Pokrovsky-Talapov transition [9]) has many characteristics of ordinary second-order transitions. However, the correlation length is infinite inside the entire IC phase and the density-density correlation function decays algebraically with the critical index $x=1$. Even though the correlation length is infinite, thermodynamic quantities $(d, c$, etc.) are not singular except at the transition. There are other length scales (than correlation lengths) which are responsible for the behaviour of the specific heat near the transition, i.e. the horizontal and vertical length scales, $l_{\mathrm{h}}$ and $l_{\mathrm{v}}, l_{\mathrm{h}}$ represents the average domain wall separation $(=1 / d)$ and $l_{\mathrm{v}}$ the average vertical distance between collisions of domain walls. Near the transition these length scales diverge with different exponents and the system becomes very anisotropic [10]. In fact, $l_{\mathrm{h}} \sim\left(\tilde{\mu}-\tilde{\mu}_{\mathrm{c}}\right)^{-1 / 2}$ and $l_{\mathrm{v}} \sim\left(\tilde{\mu}-\tilde{\mu}_{\mathrm{c}}\right)^{-1}$.

Because the vertical and horizontal length scales diverge as $l_{\mathrm{v}} \propto l_{\mathrm{h}}^{\tau}(\tau=2$ in this case), one can expect the anisotropic scaling $[10,11]$ of the singular part of the free energy in two dimensions to go as

$$
f\left(\mu, N^{-1}, M^{-1}\right)=b^{-(1++)} f\left(b^{y_{\mu}} \mu, b N^{-1}, b^{r} M^{-1}\right)
$$

where the reduced chemical potential $\mu=a\left(\tilde{\mu}-\tilde{\mu}_{\mathrm{c}}\right)$ and $a$ is a non-universal arbitrary metric factor, $N(M)$ is the horizontal (vertical) size of the system, and $b$ is the scaling 
factor. A parameter $r \equiv N^{\tau} / M$ is invariant under the above anisotropic scaling. So instead of the aspect ratio $s=N / M$, one should keep $r$ fixed towards the thermodynamic limit in order to get sensible results.

In the thermodynamic limit, the above equation leads to

$$
f \sim|\mu|^{(1+\tau) / y_{\mu}} .
$$

Comparing with the exact result [6], we find that $(1+\tau) / y_{\mu}=3 / 2$ or $y_{\mu}=2$ when $\tau=2$. The Fss behaviour along the renormalization group trajectory $\left(\mu N^{y_{k i}}\right.$ or $\mu M^{y_{\mu} / \tau}=$ constant), can be also deduced from (1) and the free energy behaves

$$
f \sim N^{-(1+\tau)} \quad \text { or } \quad f \sim M^{-(1+\tau) / \tau} \text {. }
$$

The domain wall density $d$ and the specific heat $c$ behaves

$$
\begin{array}{lll}
d \sim N^{y_{\mu}-(1+\tau)} & \text { or } & d \sim M^{\left[y_{\mu}-(1+\tau)\right] / \tau} \\
c \sim N^{2 y_{\mu}-(1+\tau)} & \text { or } & c \sim M^{\left(2 y_{\mu}-(1+\tau)\right] / \tau}
\end{array}
$$

These Fss exponents are confirmed in a generalized dimer model [12] where the Pokrovsky-Talapov transition occurs.

In this paper we study surface effects on the FSs behaviour of thermodynamic quantities in the anisotropic-scaling regime. We take free boundary conditions to generate surface terms. Domain walls can enter or exit at the boundary (see figure 2). These boundary conditions were shown to be very useful in numerical investigations of IC phases $[6,8]$. With these boundary conditions one can remove the complicated size-dependent factor ( $\kappa$-effect [13]) in the universal scaling term of finite-size corrections to the free energy. Then it becomes much easier to extrapolate finite-size data to the thermodynamic limit $[6,13]$. This is especially important near the $c-1 \mathrm{C}$ transition where the domain-wall density almost vanishes. In finite systems with free boundary conditions, some domain walls get pinned at the boundary and their fluctuations contribute to the surface free energy. Because there are almost no domain walls deep inside the system near the transition, it is not unusual to see strong surface effects there. We study how these surface effects influence the Fss behaviour of the free energy and the domain wall density near the transition.

First we examine the scaling relation in (1). Replacing $b$ by $N$, the singular part of the free energy can be written

$$
f=N^{-(1+\tau)} Y\left(N^{y_{\mu} \mu}\right)
$$

where $Y(x)=f(x, 1, r)$. The scaling function $Y(x)$ is expected to be universal and depends only on the shape of geometries $\left(r=N^{\tau} / M\right)$ and boundary conditions. For large $N$ and non-zero $\mu$ (large $x$ ), we expect the singular part of the free energy to have the form

$$
f=f^{(b)}+\frac{f^{(s)}}{N}+\ldots
$$

where $f^{(b)}$ and $f^{(s)}$ represent the singular parts of the bulk and surface free energy, respectively. Therefore the scaling function $Y(x)$ must be expanded for large $x \dagger$

$$
Y(x)=y_{2} x^{p_{2}}+y_{1} x^{p_{1}}+\ldots
$$


where $p_{2}=(1+\tau) / y_{\mu}$ and $p_{1}=\tau / y_{\mu}$. Note that both $y_{2}$ and $y_{1}$ are universal because $Y(x)$ is universal. Using this expansion in (5), we recover the correct bulk free energy, (2) and find the surface free energy

$$
f^{(s)}=y_{1} \mu^{\tau / y_{\mu}}+\ldots
$$

Notice that the leading exponent, $\tau / y_{\mu}$, becomes integral in our case where $\tau=y_{\mu}=2$.

Logarithmic singularities usually arise in systems with integral critical exponents [14]. The two-dimensional Isirg model is a typical example. There one has a vanishing critical exponent $\alpha$ which is responsible for the logarithmic divergence of specific heat. Phenomenologically what happens is that as $\alpha$ approaches zero, poles develop in both the coefficients of singular and regular parts of the free energy density, then cancellation of these poles generates the logarithmic term $[4,14]$. Such a mechanism can be invoked here to generate a logarithmic singularity in the surface free energy.

The standard procedure is to perturb away from this tiling model so that the values of the critical exponents change slightly [4]. The total free energy for the perturbed system is

$$
f_{\text {total }}=N^{-3} Y\left(N^{y^{\prime}{ }_{\mu} \mu}\right)+f_{r}^{(b)}+\frac{f_{r}^{(s)}}{N}+\ldots
$$

where $y_{\mu}^{\prime}=2 /(1-\varepsilon)$ and $\varepsilon$ is a small parameter. $f_{r}^{(b)}$ and $f_{r}^{(s)}$ are the regular parts of the bulk and surface free energy, respectively. The expansion of $Y(x)$ as in (7) yields the singular part of the surface free energy

$$
f_{\varepsilon}^{(s)}=y_{1} \mu^{1-\varepsilon}+\ldots
$$

The regular part of the surface free energy can be also expanded near $\mu=0$

$$
f_{r}^{(s)}=f_{0}+f_{1} \mu+\ldots
$$

where $f_{0}$ and $f_{1}$ are non-universal constants. As we let $\varepsilon \rightarrow 0$ and recover our original tiling model, we expect poles in the coefficients $y_{1}$ and $f_{1}$ to develop. These poles must cancel with each other, so we have

$$
\begin{aligned}
& y_{1}=-\frac{u}{\varepsilon}+\bar{y}_{1}+\mathcal{O}(\varepsilon) \\
& f_{1}=+\frac{u}{\varepsilon}+\bar{f}_{1}+\mathcal{O}(\varepsilon)
\end{aligned}
$$

where $u$ and $\bar{y}_{1}$ are universal because $y_{1}$ is universal. Combining the singular and regular part of the surface free energy and taking the $\varepsilon \rightarrow 0$ limit, we obtain the total surface free energy for our tiling model

$$
f_{\text {surf }}=f_{0}+q \mu+u \mu \ln \mu+\ldots
$$

where $q=\bar{f}_{1}+\bar{y}_{1}$ is a constant. Note that the coefficient of the logarithmic term, $u$, is universal. However there is a non-universal metric factor $a$ (see equation (1)) involved in the definition of the scaling field $\mu$. In this paper, we set $a=1$.

Domain wall density can be obtained by taking the derivative of (9) with respect to $\mu$. Along the renormalization group flow path (constant $x=N^{2} \mu$ ) we find

$$
d=\frac{A+B \ln N}{N}+\ldots
$$

where $A$ depends on paths (values of $x$ ) but $B=2 u$ is universal and independent of paths. The domain wall density is proportional to $1 / N$ as expected in (4) but it also 
has a logarithmic correction with a universal coefficient due to the logarithmic singularity of the surface free energy.

We test our scaling argument numerically. The FSS exponents of the free energy and the domain wall density in (3) and (4) are calculated employing the transfer matrix method. We also check the form of the surface free energy and the domain wall density in (13) and (14). Consider two semi-infinite-strip geometries: $(a)$ infinitely long in the vertical direction, but finite with free boundary conditions in the horizontal direction ( $M=\infty, N=$ finite) (figure 2), (b) infinitely long in the horizontal direction, but finite with periodic boundary conditions in the vertical direction $(M=$ finite, $N=\infty)$. This geometry is basically a $90^{\circ}$-rotation of $(a)$. Domain walls run vertically so that their average direction is parallel to the boundary of geometry $(a)$ while perpendicular in the case of $(b)$. Note that exact solutions for finite-size lattices are known for the geometry $(a)$ if periodic boundary conditions are taken instead of free boundary conditions [6].

As in our previous work [6], we set up the transfer matrix and find the largest eigenvalue and the corresponding eigenvector. The free energy is equal to the logarithm of the largest eigenvalue and the domain wall density can be obtained by sandwiching the domain wall operator between the eigenvectors. For geometry $(b)$ we calculate the free energy $f_{M}$ and the domain wall density $d_{M}$ at $\mu=0$ (the transition point) for system sizes $M=2,4,6,8,10,12$. Both the bulk free energy and domain wall density vanish at the transition. The numerical data obtained fit very well with the power law as suggested in (3) and (4). In order to extract the exponents we construct a sequence $w_{M}=M\left(1-f_{M+2} / f_{M}\right) / 2$ for the free energy and extrapolate to the thermodynamic limit using $\theta$-algorithm [15]. We repeat this procedure for the domain wall density (see table 1). We find that the free energy and domain wall density behaves

$$
\begin{aligned}
& f \sim M^{-1.51 \pm 0.02} \\
& d \sim M^{-0.52 \pm 0.03}
\end{aligned}
$$

where the exponents agree well with those in (3) and (4) when $y_{\mu}=\tau=2$.

For geometry $(a)$ we expect the surface terms arise in the free energy, and logarithmic corrections in the domain wall density, due to the free boundary conditions. We perform our calculations at the transition $\left(x=N^{2} \mu=0\right)$ for system sizes $N$ from 1 to 18 . In order to obtain the FSS exponent for the free energy it is useful to construct a sequence $g_{N}=(N+1) f_{N+1}-N f_{N}$ so as to cancel the surface term. Then the exponent can be obtained from the sequence of $w_{N}^{\prime}=N\left(1-g_{N+1} / g_{N}\right)$ in the same way as in geometry (b) (see table 2). We find that the free energy behaves

$$
f=\frac{f_{0}}{N}+\frac{A_{0}}{N^{3.07 \pm 0.10}}+\ldots
$$

where the exponent again agrees well with the scaling prediction in (3) when $\tau=2$.

Table 1. Extrapolation of the FSS exponents for the free energy and the domain wall density in geometry $(b)$. The exponents are extrapolated using $\theta$-algorithm [15] from the sequence $w_{M}^{\prime}=M\left(1-f_{M+2} / f_{M}\right) / 2$ and $w_{M}^{\prime}=M\left(1-d_{M+2} / d_{M}\right) / 2$

\begin{tabular}{lllllll}
\hline$M$ & 2 & 4 & 6 & 8 & 10 & $\infty$ \\
\hline$w_{M}^{\prime}$ & 0.63889 & 0.91031 & 1.05520 & 1.14458 & 1.20502 & $1.51 \pm 0.02$ \\
$w_{M}^{\prime d}$ & 0.29966 & 0.39150 & 0.43295 & 0.45581 & 0.46998 & $0.52 \pm 0.03$ \\
\hline
\end{tabular}


Table 2. Extrapolation of the Fss exponent for the free energy in geometry $(a)$. The exponent is extrapolated using $\theta$-algorithm [15] from the sequence $w_{N}^{\prime}=N\left(1-f_{N+1} / f_{N}\right)$.

\begin{tabular}{llllllll}
\hline$N$ & 12 & 13 & 14 & 15 & 16 & 17 & $\infty$ \\
\hline$w_{N}^{\prime}$ & 2.47219 & 2.52626 & 2.57305 & 2.61381 & 2.64956 & 2.68110 & $3.07 \pm 0.10$ \\
\hline
\end{tabular}

For the domain wall density we use a different procedure from above to find the coefficient $B$ of the logarithmic correction in (14). First we construct a new sequence $q_{N}=\exp \left(-N d_{N}\right)$. Then the coefficient $B$ is obtained by extrapolating the sequence $B_{N}=N\left(1-q_{N+1} / q_{N}\right)$ (see table 3 ). This sequence converges nicely to $B=0.360 \pm 0.005$. As predicted in our scaling theory, this coefficient must be independent of renormalization group flow paths, i.e. $x=N^{v} \mu$. So we repeat our calculations along different paths and find that there is almost no $x$-dependence for the value of $B$ (see table 3 ). The quality of data deteriorates as $x$ becomes larger because we cannot have enough data for larger system sizes.

Even though the fitting of the domain-wall density to the form in (14) is very good, we also try the power-law fitting. Assume that the domain wall density is proportional to $N^{-\omega}$ without logarithmic corrections. Using the same technique as before, we find $\omega=0.882 \pm 0.003$ and the convergence is deceivingly good, in fact, almost as good as in the previous logarithmic fitting. Therefore we cannot ignore the possibility of the power-law decay of the domain-wall density with a new exponent $\omega$. If it turns out real, a simple scaling theory predicts that the surface free energy must have a new singular term proportional to $\mu^{(1+\omega) / 2}$. We test this possibility and our previous prediction by studying the scaling behaviour of the surface free energy near the transition.

Inside the critical incommensurate phase the free energy takes the form

$$
f_{N}(\mu)=f_{\infty}(\mu)+\frac{f_{\text {surf }}(\mu)}{N}-\frac{\pi \zeta}{24 N^{2}}+\frac{f_{3}}{N^{3}}+\ldots,
$$

where $\zeta$ is the anisotropy factor. The bulk free energy $f_{\infty}(\mu)$ and the anisotropy factor $\zeta$ are known analytically [6]. We calculate the free energy at various values of $\mu$ and system sizes $N$. Then the surface free energy $f_{\text {surf }}(\mu)$ is obtained by using a twoparameter fit in (17) and extrapolating to the thermodynamic limit. Afterwards we fit $f_{\text {surf }}(\mu)$ to the form in (13) near $\mu=0$, which yields $f_{0}=-0.57286 \pm 0.00002, q=$ $-0.22 \pm 0.04$ and $u=0.18 \pm 0.01$. One of the critical tests of our scaling theory is to

Table 3. The universal coefficient $B$ obtained by fitting the domain wall density to equation (14) along different paths, $x=N^{\prime} \mu \mu$. The sequence $B_{N}=$ $N\left(1-\exp \left(-(N+1) d_{N+1}\right) / \exp \left(-N d_{N}\right)\right)$ is stable and higher-order corrections are almost negligible. From these data we estimate $B=0.360 \pm 0.005$.

\begin{tabular}{lllllll}
\hline$N$ & 12 & 13 & 14 & 15 & 16 & 17 \\
\hline$x=0$ & 0.36070 & 0.36116 & 0.36134 & 0.36132 & 0.36115 & 0.36088 \\
$x=0.05$ & 0.36003 & 0.36053 & 0.36075 & 0.36077 & 0.36063 & 0.36039 \\
$x=0.1$ & 0.35935 & 0.35990 & 0.36016 & 0.36022 & 0.36011 & 0.35990 \\
$x=0.5$ & 0.35392 & 0.35484 & 0.35543 & 0.35578 & 0.35594 & 0.35595 \\
$x=1.0$ & 0.34703 & 0.34843 & 0.34945 & 0.35017 & 0.35066 & 0.35098 \\
\hline
\end{tabular}


check the predicted relation between two coefficients $B$ and $u(B=2 u)$. This prediction is now confirmed within numerical accuracy. The surface free energy in (13) with the above coefficients matches the numerical data almost perfectly over a range of $\mu$, i.e. $\mu=0 \sim 0.03$ (see figure 3). In contrast, the power-law form $\left(\mu^{(1+\omega) / 2}\right)$ cannot fit the numerical data over more than one fifth of the region where the logarithmic fitting is almost perfect. Therefore we exclude the possibility of the power-law form with a non-integral exponent $\omega$.

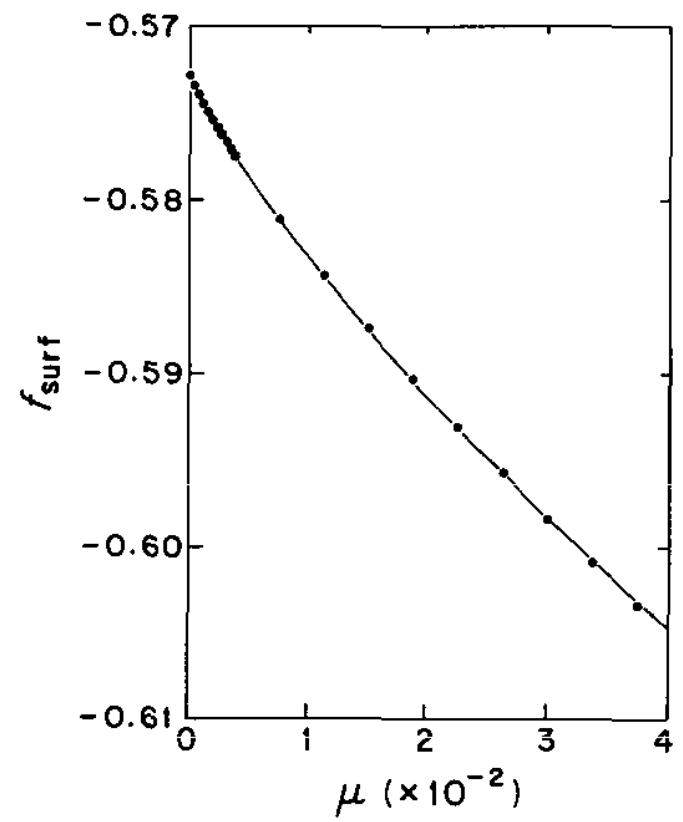

Figure 3. Plot of the surface free energy $f_{\text {surf }}$ against the reduced chemical potential $\mu\left(=\tilde{\mu}-\tilde{\mu}_{c}\right)$. The points indicate numerical data and the full line is obtained from (13) with coefficients $f_{0}=-0.57286, q=-0.22$, and $u=0.18$.

In summary, we have performed finite-size-scaling analysis for a random tiling model near the commensurate-incommensurate phase transition. The finite-size-scaling exponents can be well explained by the scaling arguments when anisotropy is taken into account. In particular, the anisotropic scaling arguments suggest an integral surface critical exponent, which implies a logarithmic singularity in the surface free energy near the transition. This logarithmic singularity is manifested in the finite-size corrections to the domain wall density. We confirm this numerically employing the transfer matrix method in the case of free boundary conditions. Finally we remark that the same behaviour was observed in the free fermion model which belongs to the same universality class [16].

\section{Acknowledgments}

We wish to thank $\mathrm{M}$ Widom for useful discussions and critical reading of this paper, and D P Deng for a valuable comment on numerical fitting. This work was supported in part by NSF grant No. DMR-8918810 and by the Donors of the Petroleum Research 
Foundation. Most of the computation was done at Pittsburgh Supercomputer Center and their service is gratefully acknowledged.

\section{References}

[1] Cardy J 1988 Phase Transitions and Critical Phenomena vol 11, ed C Domb and J L Lebowitz (London: Academic)

[2] Blöte H W Cardy J L and Nightingale M P 1986 Phys. Rev. Lett. 56742

Affleck 11986 Phys. Rev. Lett. 56746

[3] Cardy J L 1986 Nucl. Phys. B 270 [FS16] 186; 1986 Nucl. Phys. B 275 [FS17] 200

di Francesco P, Saleur H and Zuber J B 1987 J. Stat. Phys. 4957

Saluer H 1987 J. Phys. A: Math. Gen. 20 L1127

Park H and den Nijs M 1988 Phys. Rev. B 38565

Park H and Widom M 1989 Phys. Rev. Lett. 631193

[4] Privman V 1990 Finite Size Scaling and Numerical Simulation of Statistical Systems ed V Privman (Singapore: World Scientific)

[5] Binder K 1983 Phase Transitions and Critical Phenomena vol 8, ed C Domb and J L Lebowitz (London: Academic)

[6] Li W, Park H and Widom M 1990 J. Phys. A: Math. Gen. 23 L573

[7] Wang N, Chen H and Kuo K H 1987 Phys. Rev. Lett. 591010

[8] Li W, Park H and Widom M J. Stat. Phys.

[9] Pokrovsky V L and Talapov A L 1979 Phys. Rev. Lett. 4265

[10] den Nijs M 1988 Phase Transitions and Critical Phenomena vol 12, ed C Domb and J L Lebowitz (London: Academic)

[11] For a review, see K Binder and J-S Wang 1989 J. Stat. Phys. 5587

[12] Bhattacharjee S M and Nagle J F 1985 Phys. Rev. A 313199

[13] Park H and Widom M 1990 Phys. Rev. Lett. 64 1076; 1990 J. Stat. Phys. 61

[14] Wegner F 1972 Phys. Rev. B 54529

Chase S I and Kaufman M 1986 Phys. Rev. B 33239

Privman V and Rudnick J 1986 J. Phys. A: Math. Gen. 19 L1215

[15] Barber M N, Peschel I and Pearce P A 1984 J. Stat. Phys. 37497

[16] Deng D P, Park H and Widom $M$ unpublished 